Nervenarzt $2010 \cdot 81: 5-6$

DOI 10.1007/s00115-009-2899-6

C) Springer-Verlag 2010

\author{
F. Schneider \\ Klinik für Psychiatrie und Psychotherapie, Universitätsklinikum Aachen
}

\title{
Bildgebung in der Psychiatrie
}

sprechend der Fragestellung einzusetzen. Während beispielsweise die MEG Schwächen in der Lokalisation der Signalquellen hat - hier müssen Modellannahmen bei der Analyse in das statistische Modell einfließen -, hat sie unbestreitbar Vorteile aufgrund ihrer Schnelligkeit. Sie erlaubt die Erfassung von Veränderungen im Bereich von Millisekunden. Mithilfe der funktionellen MRT sind dagegen mittlerweile weitgehend hypothesenfreie Analysen möglich, die von ihr erfassbaren Reaktionen sind aufgrund der zugrunde liegenden Änderungen im Blutfluss aber deutlich langsamer. Die PET wiederum ermöglicht sehr gute quantitative Aussagen, ist aber aufgrund der radioaktiven Belastung nur sehr eingeschränkt für wiederholte Untersuchungen geeignet. mographen oder einer PET-Kamera zur Verfügung, die Magnetoenzephalographie (MEG) ist dagegen noch nicht so verbreitet. Ein Problem ist die Verfügbarkeit dieser Methoden, die bislang nur an wenigen Klinika wie Aachen mit eigenem 3-Tesla-MR und eigener PET-Kamera im unmittelbaren Bereich der psychiatrisch-psychotherapeutischen Kliniken angesiedelt ist. Mit der zunehmenden Bedeutung des Neuroimagings im Rahmen der Forschung wie der klinischen Versorgung wird eine Aufstellung dieser Geräte in Trägerschaft und Verantwortlichkeit der psychiatrischen Kliniken immer wichtiger, um nicht weiter abhängig von den jetzigen Betreibern sein zu müssen. Dabei sind Kooperationsmodelle, wie schon jetzt an einigen Standorten erfolgreich etabliert, durchaus akzeptabel, wenn ein kollegialer Austausch erfolgt.

Für die Zukunft wird es von besonderer Bedeutung sein, die Vorteile und Einschränkungen der verschiedenen Verfahren zu verstehen und sie sachgerecht ent-

\section{( $)$ Ziel ist es, Vorteile und Einschränkungen der verschiedenen Verfahren zu verstehen}

Mit diesem Sonderheft Der Nervenarzt wollen wir den aktuellen Stand der Bedeutung dieser Verfahren für die Psychiatrie und Psychotherapie diskutieren. AuBerdem wollen wir den Blick etwas erweitern auf die angrenzenden Themenbereiche Neurogenetik und Metaanalysen von Bildgebungsuntersuchungen.

Die besonderen Charakteristika der MEG und insbesondere deren Unterschiede und Vorteile gegenüber der Elektroenzephalographie (EEG) stehen zu Beginn. Klaus Mathiak und Kollegen stellen anhand verschiedener experimenteller Paradigmen heraus und machen nachvollziehbar, dass die MEG aufgrund ihrer hervorragenden zeitlichen Auflösung und Sensitivität insbesondere geeignet ist, Stö- rungen der Informationsverarbeitung im visuellen oder auditorischen System abzubilden, hierauf aber nicht beschränkt ist. Ihre Relevanz beweist sie aber fortwährend insbesondere bei Untersuchungen mit Patienten mit Schizophrenie oder Depression.

Die fMRT hat sich in den vergangenen Jahren zu einer der am meisten verwendeten Methoden entwickelt, dies ist auf die vergleichsweise einfache Anwendung und die geringen Risiken der Methode zurückzuführen. Jeder, der sich für psychiatrische Forschung interessiert, wird immer wieder mit Ergebnissen aus funktionellen MRT-Studien konfrontiert, daher stellen Birgit Derntl und Kollegen nicht nur gleichermaßen Vor- und Nachteile der fMRT vor, sondern erläutern kurz deren physikalische Grundlage, den BOLDEffekt. Der Beitrag geht außerdem näher auf Messsequenzen, Paradigmen und die Auswertung von fMRT-Daten ein. Schließlich werden exemplarische fMRTStudien aus dem Bereich der Empathieforschung dargestellt. Diese dienen vor allem der Verdeutlichung der Möglichkeiten der Methode und der Interpretation ihrer vorrangig korrelativen Ergebnisse. Insbesondere bei Untersuchungen zu emotionalen und kognitiven Beeinträchtigungen psychisch Erkrankter hat die fMRT unser Wissen und Verständnis erheblich verbessert. Für die Zukunft sind auf dem Gebiet der fMRT spannende Weiterentwicklungen zu erwarten, die die Methode hoffentlich zu einem klinisch tauglichen Diagnostikum oder sogar Therapeutikum machen.

In der jüngeren Vergangenheit wurde in der psychiatrischen Forschung große Hoffnung auf das Verständnis möglicher genetischer Ursachen gesetzt. Zunehmend 
stellt sich aber heraus, dass die Varianzaufklärung einzelner genetischer Untersuchungen in Bezug auf die Erkrankungswahrscheinlichkeit für eine bestimmte psychische Erkrankung eher begrenzt ist. Daher ist naheliegend, die beiden „Königswege“" psychiatrischer Forschung, die Molekulargenetik und die Bildgebung, zu verbinden: So stellen Udo Dannlowski und Mitarbeiter die Verknüpfung genetischer und bildgebender Studien dar. Die Autoren geben außerdem einen detaillierten Überblick über bisherige Untersuchungsergebnisse genetischer Einflüsse auf die Neurobiologie der Emotionsverarbeitung. So können bildgebende Befunde, z. B. bei depressiv erkrankten Patienten, als Endophänotypen genetischer Prädispositionen identifiziert werden.

\section{(2) Bildgebende Verfahren sind aus der Psychiatrie nicht mehr wegzudenken}

Die Ergebnisse bildgebender Untersuchungen sind in ihrer Aussagekraft oft aufgrund relativ kleiner Stichprobengrößen begrenzt. Funktionelle MRT-Studien in der psychiatrischen Forschung werden heutzutage mit Stichproben ab $12 \mathrm{~Pa}$ tienten veröffentlicht, eine Zahl, die sich in einer Art Konsens als Mindestanforderung entwickelt hat. Eine so geringe Stichprobe, insbesondere wenn sie mit einer nicht allzu scharfen Definition der Untersuchungsgruppe gekoppelt ist, erlaubt aber nur sehr pauschale Aussagen; notwendige und wichtige Folgeanalysen, z. B. von Subgruppen (anhand des Krankheitsverlaufs oder geschlechtsspezifisch), sind oft nicht möglich. Neben der Chance, größere Stichproben in MulticenterStudien zu rekrutieren, finden sich deshalb in letzter Zeit zunehmend metaanalytische Zusammenfassungen von bildgebenden Befunden. Simon Eickhoff und Kollegen stellen in ihrem Beitrag Metaanalysen als Werkzeug der bildgebenden Hirnforschung vor und erläutern deren konzeptuelle Grundlagen, Möglichkeiten und Einschränkungen. Die Autoren betonen den großen Nutzen von Metaanalysen in der psychiatrischen Forschung und zeigen, welchen Beitrag diese zum besseren Verständnis allgemeiner Pathomechanismen leisten können.
Selbstverständlich wäre ein Heft zu dem Thema „Bildgebung in der Psychiatrie" unvollständig ohne eine Darstellung der Positronenemissionstomographie (PET). Diese Methode ist sicher für das Verständnis der neurochemischen Grundlagen unseres Gehirns und insbesondere der Wirkung bekannter und neuer Psychopharmaka unverzichtbar. Die Autoren Gerd Gründer, Ingo Vernaleken und Peter Bartenstein stellen daher die Anwendungen der PET und der Single-Photon-Emissions-Computertomographie (SPECT) im Bereich der Psychiatrie dar. Die Autoren fassen die wichtigsten Befunde aus verschiedenen Untersuchungen bei psychiatrischen Patienten zusammen und verdeutlichen das enorme Potenzial von PET-Studien in Bezug auf neue Erkenntnisse zu Rezeptor- und Transportereigenschaften sowie der pharmakologischen Wirksamkeit von Medikamenten. Dieser Beitrag wurde als CME-Beitrag gestaltet, nicht zuletzt um auch zu betonen, dass ein Verständnis bildgebender Verfahren in unserem Fachgebiet als Basiswissen anzusehen ist.

Ich würde mich sehr freuen, wenn Sie dieses Heft und die Beiträge mit einem besonderen Interesse aufnehmen und dass Sie meine Einschätzung teilen, dass bildgebende Verfahren aus der Psychiatrie nicht mehr wegzudenken sind und in $\mathrm{Zu}$ kunft noch an Bedeutung zunehmen werden. Zum Verständnis psychischer Erkrankungen sind diese Methoden natürlich keineswegs als einzige Vorgehensweisen zu verstehen. Die besondere Chance, die sich der Psychiatrie eröffnet, liegt in dem breiten Spektrum der derzeit verfügbaren, bezahlbaren und vom Risikoprofil beherrschbaren Methoden, immer in Verbindung zu unseren traditionellen psychopathologischen Methoden. Neben den Methoden der Bildgebung sind dies neben epidemiologischen Ansätzen natürlich auch Vorgehensweisen in der Neurogenetik und insbesondere auch in der molekularen Bildgebung. Ein wissenschaftlicher Fortschritt in unserem Fach ist insbesondere daraus zu erwarten, dass es in Zukunft zunehmend gelingen wird, weitere wichtige Informationsquellen, z. B. aus der Versorgungsforschung oder der Psychopathologie, miteinander in Einklang zu bringen. Es ist dane- ben zu erwarten, dass aus zukünftigen integrierten Ansätzen neue Krankheitskonzepte entstehen werden, die unsere derzeitige Klassifikation weiterentwickeln und vor allem neue Ideen für erfolgreiche Therapien entstehen.

In diesem Sinne wünsche ich Ihnen eine spannende Lektüre.

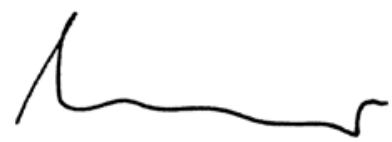

Mit kollegialen Grüßen

Frank Schneider, Aachen

\section{Korrespondenzadresse \\ Prof. Dr. Dr. F. Schneider}

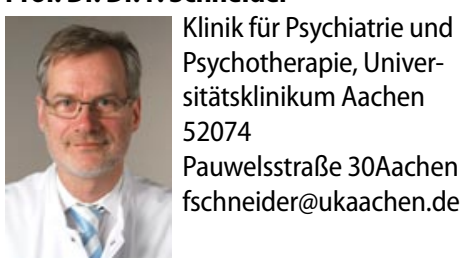

